\title{
Preliminary photometric study of a pulsating component in the eclipsing binary AB Per
}

\author{
S.-L. Kim, S.-G. Kwon ${ }^{1}$, D.J. Lee, C.-U. Lee, H. Jin \\ Korea Astronomy Observatory, Daejeon, 305-348, Korea
}

\author{
J.W. Lee \\ Department of Astronomy and Space Science, Chungbuk National \\ University, Cheongju, 361-763, Korea
}

\begin{abstract}
We present new photometric results of the Algol-type semidetached eclipsing binary AB Per which was recently discovered to have a Delta Scuti pulsating component.
\end{abstract}

Delta Scuti pulsating components in eclipsing binary systems are attractive observing targets for asteroseismology because we can identify their non-radial modes using the amplitude and phase changes during the eclipse. The masses and radii for each component in eclipsing binaries can be accurately determined to help model the pulsation frequency spectra. We have been performing a photometric survey to search for Delta Scuti pulsating components in eclipsing binary systems since 2001 September at the Sobaeksan Optical Astronomy Observatory (SOAO) in Korea, using the 61-cm telescope and a $2 \mathrm{~K}$ CCD camera. Two new pulsating components of RX Hya and AB Per among 14 observing targets have been discovered from the first year of observations (Kim et al. 2003).

We carried out the follow-up observations for the Algol-type semi-detached eclipsing binary AB Per to investigate its photometric variations in detail. The observations were obtained on 29 nights from 2002 January to 2003 March with a Johnson $B$ filter, using the SOAO 61-cm and BOAO (Bohyunsan Optical Astronomy Observatory) $1.8-\mathrm{m}$ telescopes in Korea, and the KAO 1-m telescope that was recently installed at Mt. Lemmon, Arizona, USA. We used HD 275605 $(V=10.55,(B-V)=0.48)$ as a comparison star and several other stars in the observing field as check stars. Using the IRAF package, we processed timeseries CCD frames to correct the bias level and the pixel-to-pixel inhomogeneity of quantum efficiency (flat fielding) to obtain instrumental magnitudes. After removing light variations caused by the eclipses (Fig. 1), we examined oscillation features of AB Per.

Fig. 2 displays the power spectra of the residuals after the synthetic eclipse curves have been subtracted from the data. We confirmed short-term variations of AB Per with a frequency of $5.1187 \mathrm{~d}^{-1}(P=0.19536 \mathrm{~d})$. Two low frequencies of $f_{1}=0.0034 \mathrm{~d}^{-1}$ and $f_{3}=0.3357 \mathrm{~d}^{-1}$ might result from the incomplete eclipse curve solution. The residuals are shown in Fig. 3, superimposing synthetic

\footnotetext{
${ }^{1}$ Present address: Astronomy program, School of Earth and Environment Sciences, Seoul National University, Seoul, 151-742, Korea
} 


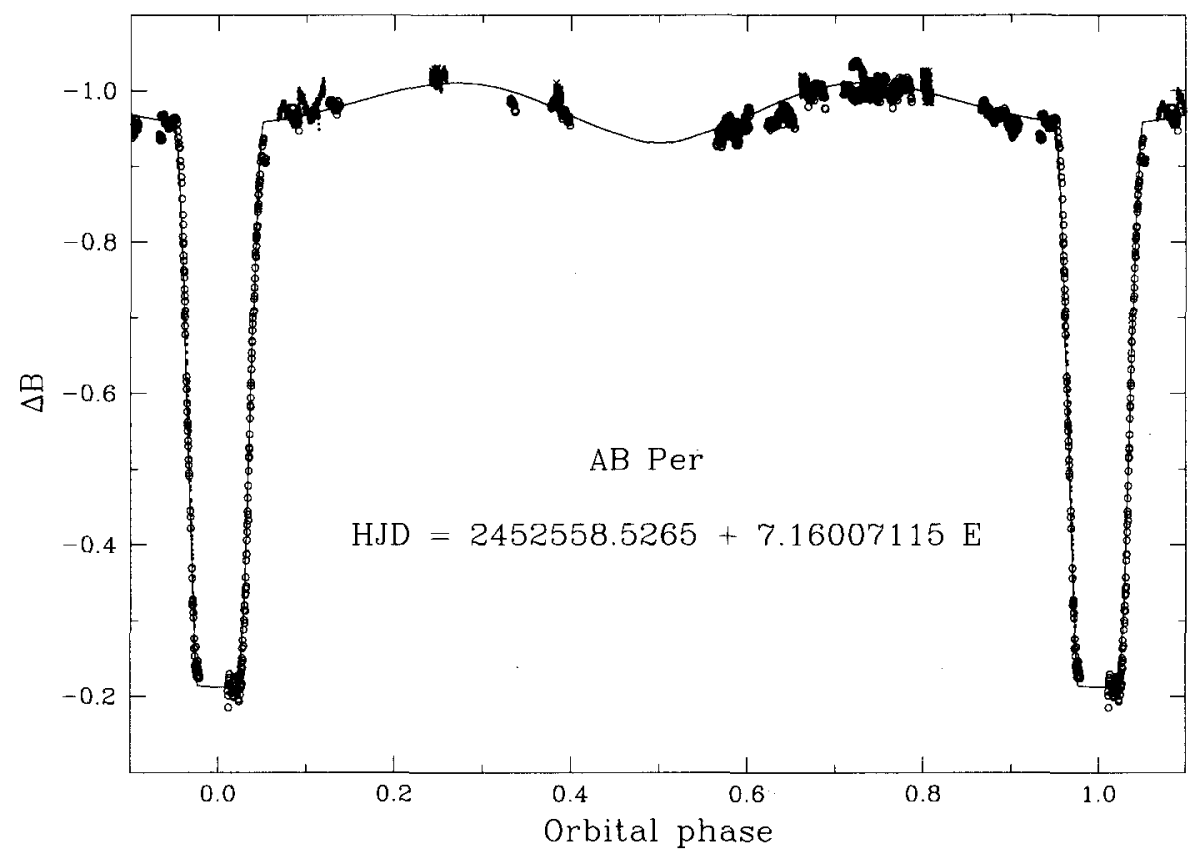

Figure 1. Orbital phase diagram of AB Per with $P_{o r b}=7.16 \mathrm{~d}$. The line represents a synthetic light curve from the Wilson-Devinney binary code. Open circles are data from $\mathrm{SOAO}$, crosses from $\mathrm{BOAO}$ and small dots from Mt. Lemmon.

curves obtained from the frequency analysis. There are two possible mechanisms for these short-term variations: Delta Scuti pulsation or rotation-induced variability (e.g. ellipsoidal variability, surface inhomogeneity, etc.). Delta Scuti pulsation is the most reasonable source of the short-period variations, given the relatively long orbital period of $7.16 \mathrm{~d}$ and the normal synchronization scenario for EA/SD type stars (Glazunova 1999). It is highly desirable to get simultaneous photometric data with $B$ - and $I$-bands to measure color (temperature) variations caused by pulsations.

\section{References}

Kim, S.-L., Lee, J.W., Kwon, S.-G., Youn, J.-H., Mkrtichian, D., Kim, C. 2003, A\&A, 405, 231

Glazunova, L.V. 1999, Astronomy Letters, 25, 467 

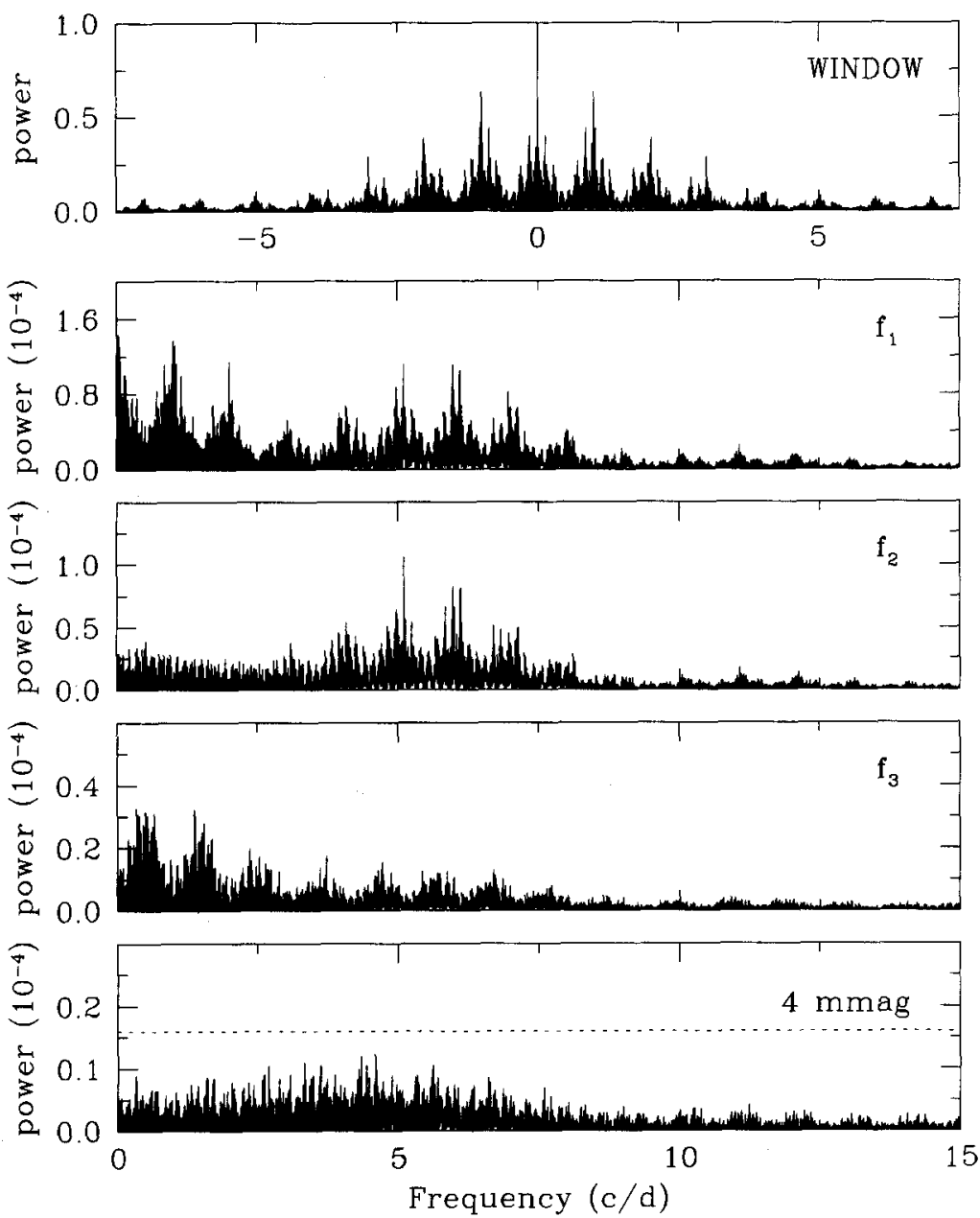

Figure 2. Power spectra for AB Per obtained from the residuals after removal of the eclipse curve. Power is in $\mathrm{mag}^{2}$. 


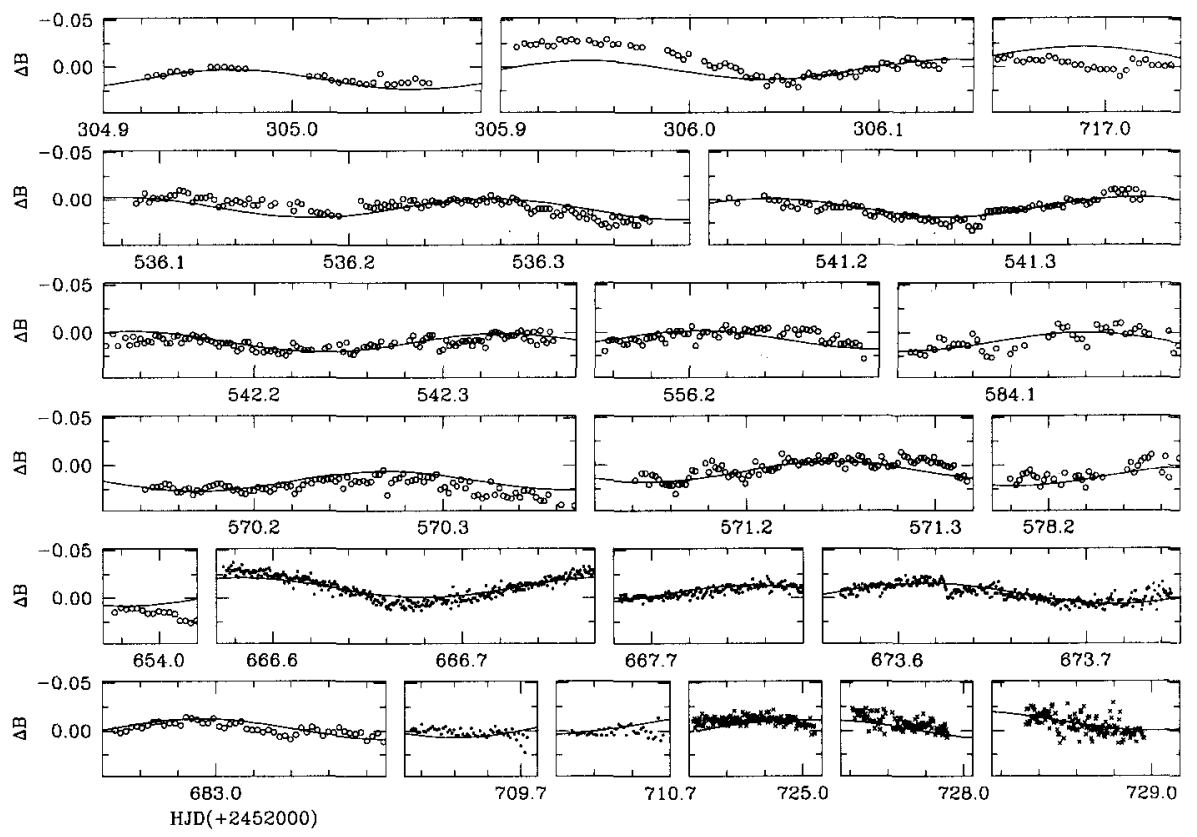

Figure 3. Light variations of the residuals for AB Per. Synthetic curves (lines) generated from the multiple frequencies determined from the frequency analysis are superimposed on the data. The data represented are the same as in Fig. 1. 